\title{
ROSAT X-ray sources in the field of the LMC ${ }^{\star}$
}

\section{Statistics of background AGN and X-ray binaries}

\author{
P. Kahabka ${ }^{\star \star}$ \\ Sternwarte, Universität Bonn Auf dem Hügel 71, 53121 Bonn, Germany \\ Received 3 September 2001 / Accepted 22 March 2002

\begin{abstract}
About 200 X-ray sources from a sample of spectrally hard ROSAT PSPC sources, given in the catalog of Haberl \& Pietsch (1999), and observed in a 60 square degree field of the LMC during several archival pointed observations with a wide range of exposure times have been reanalyzed. For these sources accurate count rates and hardness ratios have been recalculated. In comparison to Haberl \& Pietsch (1999) we used merged data from all available observations and we derived average source parameters by investigating each source individually. From a simulation powerlaw spectral tracks have been derived in the $H R 1-H R 2$ plane and $\sim 170$ sources have been classified as background X-ray sources or as LMC X-ray binaries. $80 \%$ of the spectrally hard X-ray sources with more than 50 observed counts have been found to be consistent with background X-ray sources and $20 \%$ with LMC X-ray binaries (53 sources with AGN and 15 with X-ray binaries). The discovery of a new supersoft source RX J0529.4-6713 at the southern H I boundary of the supergiant shell LMC 4 is reported. We find two new candidate X-ray binary systems which are associated with the optical bar of the LMC and additional candidate X-ray binaries which are associated with supergiant shells.
\end{abstract}

Key words. galaxies: Magellanic Clouds - galaxies: individual: LMC - galaxies: active - galaxies: ISM $\mathrm{X}$-rays: galaxies - X-rays: stars

\section{Introduction}

X-ray background sources are active galactic nuclei (AGN) and clusters of galaxies for which the integral number versus flux distribution (the $\log N-\log S$ ) has been derived for different fields in the sky (cf. Hasinger et al. 1998; Gilli et al. 1999; Gilli et al. 2001). In the direction of nearby galaxies additional bright and spectrally hard X-ray sources are discovered which are associated with X-ray binaries (cf. for M 31 Supper et al. 2001 and for M 33 Haberl \& Pietsch 2001). In the direction of the Magellanic Clouds which cover an area of a few 10 square degrees a few 100 background X-ray sources have been detected in deep pointed observations (cf. Haberl \& Pietsch 1999a; Haberl et al. 2000). The $\sim 20-30$ pulsating X-ray sources discovered in the Magellanic Clouds are to a large fraction associated with Be-type X-ray binaries (e.g. Liu et al. 2000). In addition a few bright blackhole X-ray binaries (LMC X-1 and LMC X-3) exist in the LMC. In an investigation of the ROSAT all-sky survey (RASS) observations of a $13^{\circ}$ by $13^{\circ}$ field centered on the LMC, more than

\footnotetext{
* Tables 1 and 3 are only available in electronic form at the CDS via anonymous ftp to

cdsarc.u-strasbg.fr $(130.79 .128 .5)$ or via

http:/cdsweb.u-strasbg.fr/cgi-bin/qcat?J/A+A/388/100

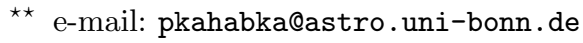

500 X-ray sources have been found (Pietsch \& Kahabka 1993). The ROSAT all-sky survey covered a large field of the LMC area and was only in regions close to the elliptical pole (north east of the LMC disk) deep enough to allow a detailed analysis of X-ray sources. Making use of the RASS faint source catalog (Voges et al. 2000) 1200 sources are found within about the same investigated field. For this sample a limiting flux of $1.3 \times 10^{-13} \mathrm{erg} \mathrm{cm}^{-2} \mathrm{~s}^{-1}$ is derived assuming a galactic foreground absorbing column of $5 \times 10^{20} \mathrm{~cm}^{-2}$ and a count rate to flux conversion factor for a powerlaw photon spectrum with $-\Gamma=2.0$. A much deeper survey of the LMC field has been performed during pointed ROSAT PSPC (and HRI) observations. A catalog of ROSAT PSPC X-ray sources in a $10^{\circ}$ by $10^{\circ}$ field of the LMC has been established by Haberl \& Pietsch (1999a). The catalog comprises $758 \mathrm{X}$-ray sources and identifications are given for 144 sources. In addition time variability has been taken into account by Haberl \& Pietsch (1999b) to refine the classification of $\sim 15$ LMC X-ray sources. For this sample, derived in the deeper pointed observations of the LMC field, a limiting flux of $4 \times 10^{-15} \mathrm{erg} \mathrm{cm}^{-2} \mathrm{~s}^{-1}$ is derived. (Subsequently it is referred to the Haberl \& Pietsch 1999a and 1999b papers as HP99). A catalog of ROSAT HRI X-ray sources in a $10^{\circ}$ by $10^{\circ}$ field of the LMC has been established by Sasaki et al. (2000, hereafter SHP00). They found $397 \mathrm{X}$-ray sources of which 259 are 
new detections in addition to the ROSAT PSPC X-ray sources found by Haberl \& Pietsch. A fraction of the classifications of the ROSAT sources in the LMC field are not firm partly due to lacking optical identifications and partly due to X-ray characteristics which allow more than one source class.

I reanalyzed a considerable fraction of the sample of classified and unclassified spectrally hard X-ray sources in the LMC field and given in the ROSAT PSPC catalog of HP99. I combined (merged) the observational data of these sources which have been obtained when the source was at ROSAT PSPC off-axis angles of up to $50^{\prime}$. In general the analysis has been restricted to observations where the source was at an off-axis angle of $\lesssim 30^{\prime}$. I give in Sect. 2 the selection criteria for the sample of spectrally hard sources. I will derive in Sect. 3 the basic properties of this reanalyzed sample. I especially derive count rates in the spectrally hard (0.5-2.0 keV) and broad (0.1-2.4 keV) band as well as the X-ray colors (hardness ratios HR1 and HR2). For few sources positions more accurate than given in HP99 are derived. In Sect. 4 I derive from simulations tracks for powerlaw spectra in the $H R 1-H R 2$ plane. It is made use of these tracks to achieve a classification of the spectrally hard sources as AGN or as X-ray binaries. I also take into account the AGN sample for which X-ray spectral fitting has been performed in Kahabka et al. (2001, Paper I). In addition I constrain in Sect. 5 for the classified background X-ray sources the hydrogen absorbing column density due to LMC gas assuming constraints on the powerlaw photon index for AGN type spectra. For a sub-sample of candidate AGN and X-ray binaries I also derive constraints on the metallicity of the LMC gas. In Sect. 6 the X-ray binary sample is discussed and the number of X-ray binaries derived for the LMC is compared with the number of X-ray binaries observed in the SMC. Finally, in Appendix A the catalog of X-ray sources in the field of the supergiant shell LMC 4 will be given.

\section{Selection of the AGN and X-ray binary sample}

The sample of spectrally hard sources has been taken from the catalog of HP99. It comprises the sources which have already been identified by HP99 with AGN, quasars and galaxies. This sample is largely the sample which has been used in Paper I for X-ray spectral fitting. A few sources have less than $\sim 100$ counts and no X-ray spectra have been fitted for these sources. In addition, sources classified as [hard] by HP99 have been investigated. Source and background spectra have been created and from these data hardness ratios have been determined. These hardness ratios are for the ROSAT PSPC defined as

$H R 1=\frac{(\text { Hard }- \text { Soft })}{(\text { Hard }+ \text { Soft })}$

$H R 2=\frac{(\operatorname{Hard} 2-\operatorname{Hard} 1)}{(\operatorname{Hard} 1+\operatorname{Hard} 2)}$

They are determined from the counts in the standard ROSAT PSPC bands Soft $=$ (channel 11-41,
0.1-0.4 keV), Hard = (channel 52-201, 0.5-2.0 keV), $\operatorname{Hard} 1=$ (channel 52-90, 0.5-0.9 keV) and Hard $2=$ (channel 91-201, 0.9-2.0 keV). The location of a source in the $H R 1$ - HR2 plane has been used for a source classification: In case the source was located in the AGN band $-\Gamma=(2.0-3.0)$ it was classified as an AGN. The source has been classified as an X-ray binary in case it was "above" the AGN band (in the regime of spectra with less steep powerlaw indices). Not all sources classified as [hard] by HP99 are consistent with AGN or X-ray binaries (some sources are extended).

This sample has been extended by taking sources from the HP99 catalog into account which have been selected using criteria on the hardness ratios $H R 1$ and $H R 2$ and in addition on the extent likelihood ratio $M L_{\text {ext }}$ (the extent likelihood ratio equals $M L_{\text {ext }}=-\ln (P)$, with $P$ the probability that the measured photon distribution deviates from the instrument point-spread function). These criteria are: $H R 1>0.5, H R 2>0.1, H R 2-\delta H R 2<0.4$ and $M L_{\text {ext }}<30$ (for AGN) and $H R 1>0.5, H R 2-\delta H R 2>0.4$ and $M L_{\text {ext }}<30$ (for X-ray binaries). Only sources which have been observed in the inner $20^{\prime}$ of the detector have been used for the analysis. This selection may not be complete as AGN with very low absorbing columns (e.g. located in the outskirts of the LMC) are not necessarily considered. Therefore additional ROSAT PSPC sources have been investigated which were inside the $20^{\prime}$ radius of the PSPC detector and which had at least 100 counts in the broad spectral band. For these sources a source and a background spectrum have been created and from these data hardness ratios have been calculated. Taking the value of the galactic absorbing column and the low LMC absorbing column into account the location of these sources in the $H R 1$ - HR2 plane has been used to classify these sources. There were 141 sources which were classified as AGN based on this selection. The X-ray binary sample comprises 30 sources and is discussed in more detail in Sect. 6.

\section{Determination of the basic source parameters}

The basic source parameters are the coordinates, the count rate in the spectrally broad and hard band, the spectral colors (hardness ratios HR1 and HR2) and the amplitude of time variability. The hardness ratios will be used in a later section for a source classification. The count rate is used to construct the $\log N-\log S$ relation. As the standard $\log N-\log S$ uses the count rate in the hard band it is of importance that this count rate is made available. I note that usually only the count rate in the broad band is given in catalogs. Conversion from one to another is in principle possible (from simulations) but then one has to assume the proper spectral index which is a priori not known. I recalculated the count rates and the hardness ratios for a subsample using the merged data. The values which are given are the mean values integrated over all observations. I applied the EXSAS spectral fitting task (cf. Zimmermann et al. 1994) for binning and instrument 
correction (vignetting and dead time). I have individually chosen a circular region for the background subtraction which was in general close to the source and had the same size as the source circle. This procedure allowed precise values to be derived and the statistical errors to be minimized. It has to be noted that values for the hardness ratios $H R 1$ and $H R 2$ which are larger than 1.0 are possible in case the source has a negligible flux in the $0.1-0.5 \mathrm{keV}$ band and background subtraction results in a negative count rate for this band. Still consideration of the errors in the hardness ratios for such cases allows values for the hardness ratios which are consistent with 1.0 (in Figs. 1, 3-5 we present or consider sources with hardness ratios $H R 1>1.0$ and $H R 2>1.0$ in the following way: We set $H R 1=1.0$ and $H R 2=1.0$ and we show the error bar towards the minimum value).

In general the source coordinates have not been recalculated and it will be referred to the coordinates given in HP99.

\subsection{Comparison with the source parameters given in HP99}

A different approach has been chosen than has been used by HP99 to derive source parameters (count rates and hardness ratios). I have derived mean parameters from the merged data of all available observations with integration times of at least $1000 \mathrm{~s}$. This, in general, reduced the statistical errors. In addition spectra (source and source plus background) have been accumulated for each individual source and the standard instrument corrections have been applied. For time variable sources average values have been derived in the analysis. It is expected to derive more precise values for the source count rate and the X-ray colors (hardness ratios $H R 1$ and $H R 2$ ) than given in HP99. In Fig. 1 (upper panel) the broad band (0.1-2.4 keV) ROSAT PSPC count rates as derived by HP99 and in this analysis are compared. The count rate derived by these two methods follows the same trend but individual rates scatter. In addition it is found that the count rates derived by HP99 are systematically larger (by about a factor of 1.5) than the count rates derived by K01. Such a systematic difference may be explained by the different method which has been applied to determine the source counts. In addition HP99 determined the count rate of a source for the observation (in case multiple observations exist) in which the derived position was most accurate. In case of time variable sources such an approach can bias the count rate to values which are systematically larger than the mean value averaged over many observations. The importance of this effect was checked for two bright AGN in the field of the LMC (RX J0524.0-7011 and RX J0503.1-6634) which were found to be variable with a timescale of a few hundred days to few years. It was found that for these two AGN the count rate averaged over observations spread over a few years was a factor of 3 and 1.6 respectively smaller than the count rate given in the catalog of HP99.
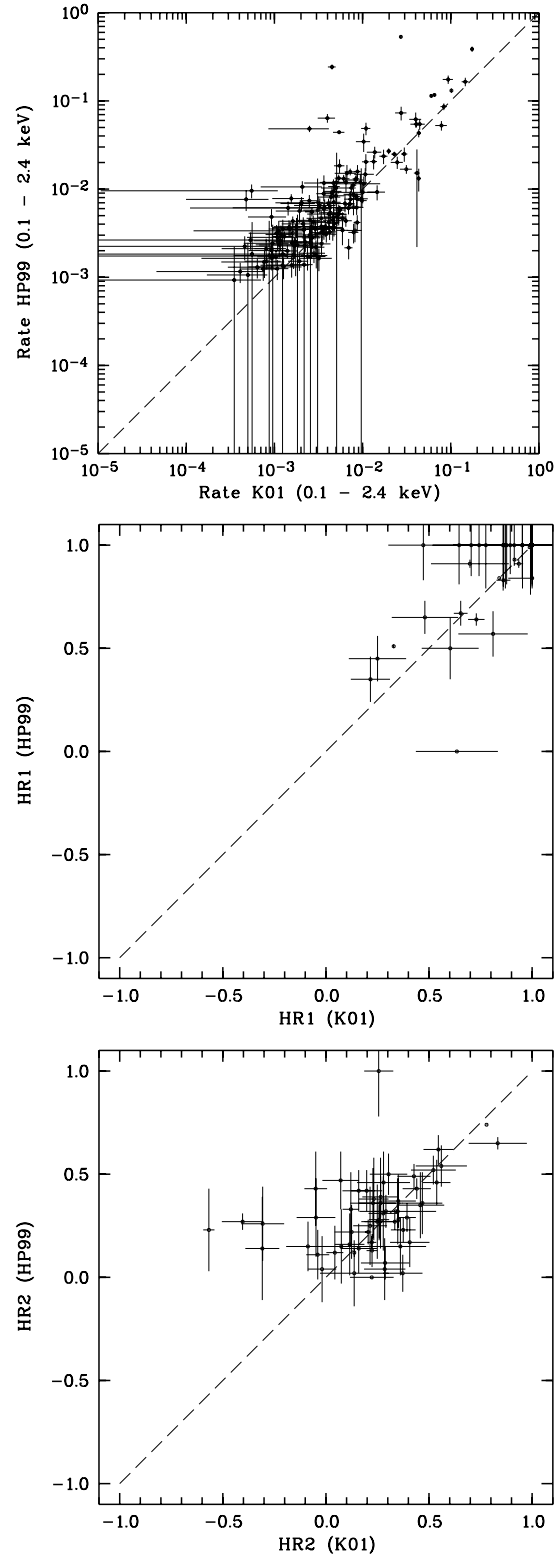

Fig. 1. Upper panel: broad band $(0.1-2.4 \mathrm{keV}) P S P C$ count rates as derived in this work (K01, Table 1) in comparison with the PSPC count rates derived by HP99. Middle panel: soft hardness ratio $H R 1$ as derived in this work (K01) in comparison with $H R 1$ derived by HP99 (only sources with $\delta H R 1<0.25$ have been plotted). Lower panel: hard hardness ratio HR2 as derived in this work (K01, Table 1) in comparison with $H R 2$ derived by HP99 (only sources with $\delta H R 2<0.25$ have been plotted). For sources with $H R 1>1.0$ and $H R 2>1.0$ a value of 1.0 is given and the error bar towards the minimum allowed value.

In addition it is found that the count rates of 17 sources deviate more than $5 \sigma$ and of 9 sources more than $10 \sigma$. 7 of the 9 sources are X-ray binaries which show large variability in the count rate with time. In the catalog presented here count rates averaged over all observations are given while in HP99 the count rate for the observation is given where the source position has been determined (the largest deviation with a few hundred sigma is found for LMC X-4, for this source a low count rate is given in the 
catalog of HP99). The remaining two sources are the AGN RX J0524.0-7011 and RX J0503.1-6634 which are variable in time (see discussion above).

In this work mainly spectrally hard sources (candidates for AGN and X-ray binaries) have been selected which have hardness ratios $H R 1$ and $H R 2>0.0$. In Fig. 1 (middle and lower panel) the comparison between the hardness ratios $H R 1$ and $H R 2$ is shown as derived by both methods for sources with precise hardness ratios $(\delta H R 1<0.25)$. There is some scatter in these values which may be explained by the different methods which have been applied. A few sources were found in this analysis to be softer than given in the catalog of HP99 (cf. the sources in the lower panel of Fig. 1 which are found at $H R 2$ values $<0$ ). These sources were not classified as AGN, cf. Table 1). In the further analysis the values for the count rate and hardness ratios derived in this work have been used.

\section{Source classification and parameter determination in the hardness ratio plane}

In Paper I the technique of X-ray spectral fitting has been applied to a sample of 26 background AGN in the field of the LMC. These AGN were taken from the ROSAT PSPC catalog of LMC X-ray sources of HP99 and candidate AGN selected here.

A different approach to constrain the spectral parameters is to use the hardness ratios $H R 1$ and $H R 2$. These hardness ratios are commonly available for ROSAT PSPC $\mathrm{X}$-ray sources published e.g. in catalogs. There are two techniques to calculate these hardness ratios. The first is to fit the ROSAT PSPC point-spread function to data binned spatially in the standard energy bands and to determine the background from a spline-fit applied to these binned data. Such a procedure can be applied to a large sample of sources detected in the field of view of a PSPC observation. The second technique is to determine the source and the background counts in the standard energy bands from observational data which have been corrected for the ROSAT PSPC instrument functions. The background counts have been derived from a spatial region close to the source.

In order to constrain the spectral parameters of a source the observed hardness ratios HR1 and HR2 have been compared with the hardness ratios determined from simulations. Such a comparison was performed with a $H R 1$ - HR2 grid derived from such simulations. Free parameters to be varied in the simulations were the powerlaw photon index $\Gamma$ of the adopted photon spectrum, the hydrogen absorbing column density $N_{\mathrm{H}}$, and the metallicity $X$. The redshift $z$ has not been varied in the simulations. It has always been set to $z=0$.

In the case of the LMC background AGN, it is expected that the parameters can be confined to well defined ranges. For example in recent work it has been found that AGN have canonical powerlaw indices which can be confined to a narrow range $-\Gamma=2.0$ to 2.5 for the ROSAT PSPC
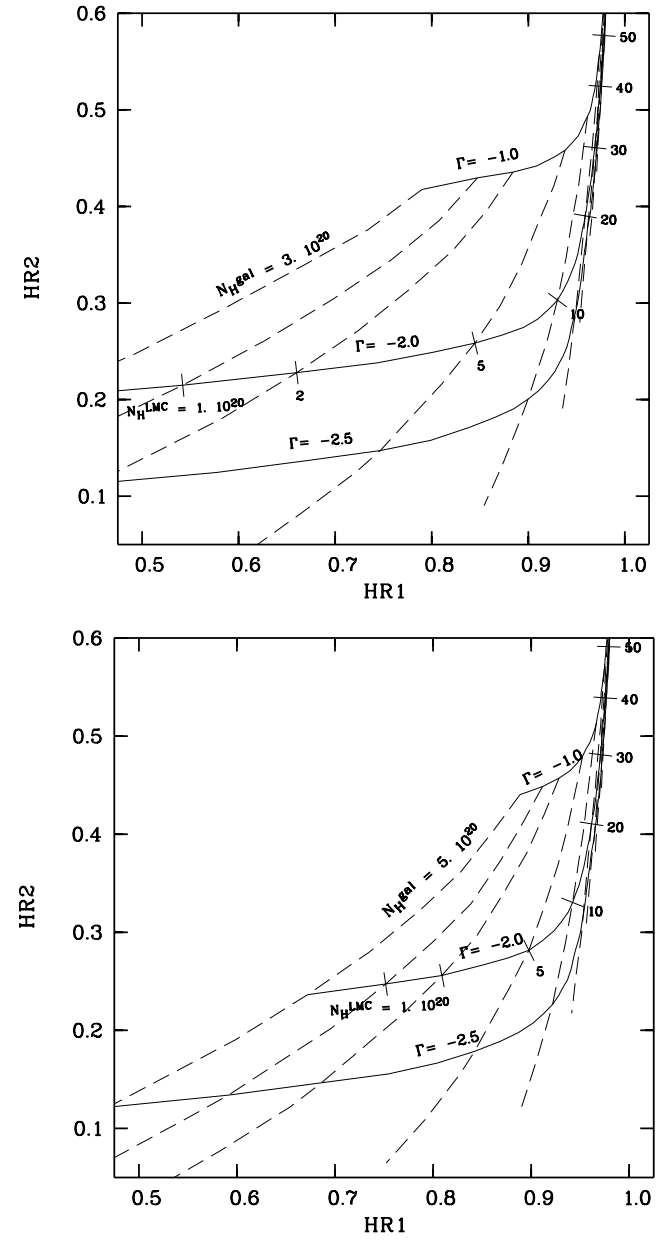

Fig. 2. Enlarged $H R 1-H R 2$ grid calculated for background $\mathrm{X}$-ray sources (AGN) and X-ray binaries, showing the effect of foreground absorption. The full lines give $\Gamma$ isolines, the dashed lines represent $N_{\mathrm{H}}$ isolines. The band for AGN type spectra is shown which is defined by $-\Gamma=(2.0: 2.5)$. The $N_{\mathrm{H}}$ isolines have been labelled at the intersection with the $-\Gamma=2.0$ line with $N_{\mathrm{H}}^{\mathrm{LMC}}$ in units of $10^{20} \mathrm{~cm}^{-2}$. The leftmost (long dashed) line is for zero LMC gas. The calculations include foreground galactic gas with $N_{\mathrm{H}}^{\text {gal }}=3 \times 10^{20} \mathrm{~cm}^{-2}$ (upper panel) and $5 \times 10^{20} \mathrm{~cm}^{-2}$ (lower panel), while for the LMC gas a mean metallicity of -0.3 dex is assumed.

(cf. Brinkmann et al. 2000). These canonical values may still somewhat depend on the chosen energy range (instrument). Also the mean metallicity of the LMC gas is quite well constrained from observational work (e.g. Dopita \& Russell 1992). These facts, in principle, allow the hydrogen absorbing column density towards an AGN from simulations to be determined. The redshift of the AGN has only a minor effect on the simulated spectra for the expected redshift range covered by the AGN sample (cf. Comastri et al. 1995). For the hydrogen column density $N_{\mathrm{H}}$ the model must account for the galactic contribution as well as the LMC contribution. These two components are assumed to have different metallicities and these models will be termed hybrid models. AGN can also show intrinsic absorption (cf. Comastri et al. 1995). But most of the intrinsically absorbed AGN will not be detected in the ROSAT 
band as the value of the absorbing column is large and the fluxes are low.

In Fig. 2 I give the $H R 1-H R 2$ grid for a hybrid $N_{\mathrm{H}}$ model. For the first component with galactic metallicity a value of $N_{\mathrm{H}}^{\text {gal }}$ of 3 and $5 \times 10^{20} \mathrm{~cm}^{-2}$ has been used, for the second component absorbing columns with mean LMC metallicities $(X=-0.3$ dex with respect to galactic interstellar absorption abundances, Morrison \& McCammon 1983) which range from $10^{20} \mathrm{~cm}^{-2}$ to $10^{22} \mathrm{~cm}^{-2}$ in steps of $10^{20} \mathrm{~cm}^{-2}$. In this classification scheme X-ray binaries cover $-\Gamma$ values of $\sim(0.5-1.6)$ and $A G N$ cover $-\Gamma$ values of $\sim(1.8-3.0)$.

The range chosen for the powerlaw photon index $\Gamma$ is the range which is presently considered to be the most reliable in the ROSAT PSPC band and is considered to be the canonical band. This range of $\Gamma$ values agrees with the range of $\Gamma$ values required in Paper I to classify AGN from the result of X-ray spectral fitting.

I have derived the values for the hardness ratios and the errors in the hardness ratios making use of the same source and background regions as chosen for X-ray spectral fitting in Paper I. The spectral data have been binned in the standard energy bands used in the hardness ratio definition and the data have been corrected using the EXSAS correction package (Zimmermann et al. 1994).

In Table 1 the catalog of the reanalyzed spectrally hard X-ray sources as taken from the catalog of HP99 is given. One additional source, RX J0536.9-6913, is contained in the catalog which is not contained in the catalog of HP99 but which has been investigated in Paper I and found to be consistent with an absorbed AGN. In the table first the sources classified as X-ray binaries are given, then the AGN, and at the end of the table a few sources classified as SNR or foreground stars are given. For the last 11 sources in the table no classification is given. In Col. 1 of Table 1 the ROSAT name is given, in Cols. 2 and 3 the source index from the same catalog and the catalog of Sasaki et al. (2000), in Cols. 4 and 5 the count rate of the broad $(0.1-2.4 \mathrm{keV})$ and hard $(0.5-2.0 \mathrm{keV})$ band, in Cols. 6 and 7 the hardness ratios $H R 1$ and $H R 2$ including $1 \sigma$ errors, in Cols. 8 and 9 the column density of the galactic and LMC H I derived from 21-cm Parkes data (Brüns et al. 2001), in Col. 10 the LMC column density derived from the hardness ratio analysis from this work, in Col. 11 the source classification, and in Col. 12 references and notes to individual sources.

\subsection{Source classification in the HR1-HR2 plane}

I made simulations in which I varied the powerlaw photon index $\Gamma$ of the source spectrum and the LMC hydrogen column density $N_{\mathrm{H}}^{\mathrm{LMC}}$ assuming reduced metallicities which are expressed with the logarithmic decrement $X$. Different values have been assumed for the powerlaw photon index $\Gamma$ for X-ray binaries and AGN $(-\Gamma=0.5$ to 1.6 for X-ray binaries and $-\Gamma=1.8$ to 3.0 for $\mathrm{AGN}$ ).
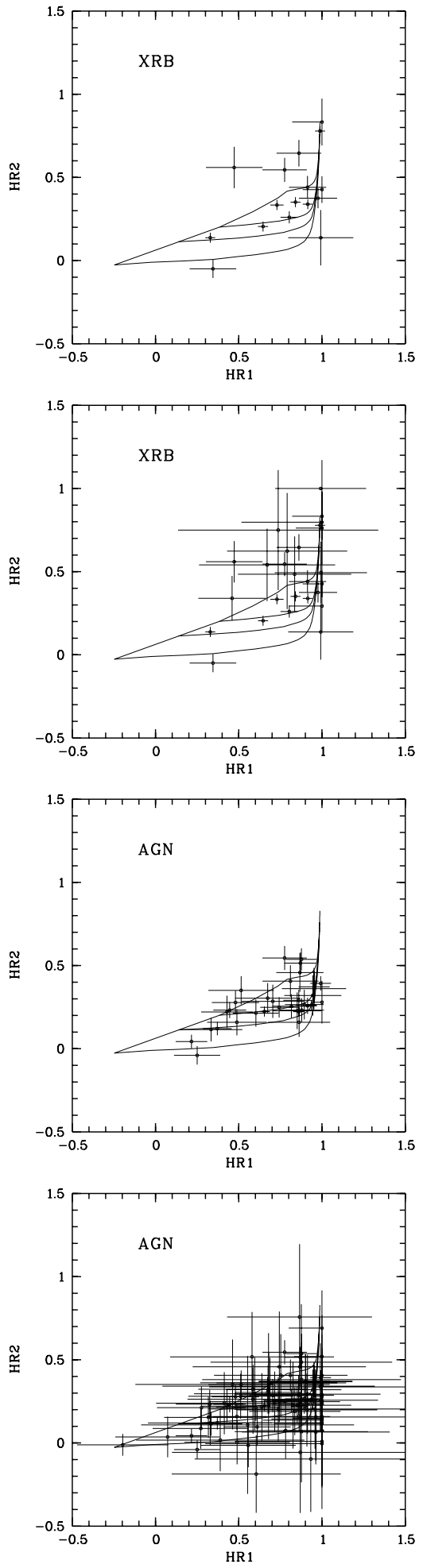

Fig. 3. Sources from Table 1 classified as X-ray binaries (XRB, upper two panels) and background AGN (lower two panels). First and third panel: sources with $\delta H R 1 \leq 0.2$ and $\delta H R 2 \leq 0.2$ are given. Second and fourth panel: sources with $\delta H R 1 \leq 0.85$ and $\delta H R 2 \leq 0.85$ are given. Also shown are the simulated (powerlaw slope $-1.0,-2.0,-2.5$, and -3.0 ) tracks for hydrogen columns in excess of galactic hydrogen columns $\left(N_{\mathrm{H}}^{\mathrm{LMC}}>0.0\right)$ and assuming a value for the galactic hydrogen column density of $N_{\mathrm{H}}^{\text {gal }}=3 \times 10^{20} \mathrm{~cm}^{-2}$. For sources with $H R 1>1.0$ and $H R 2>1.0$ a value of 1.0 is given and the error bar towards the minimum allowed value. 

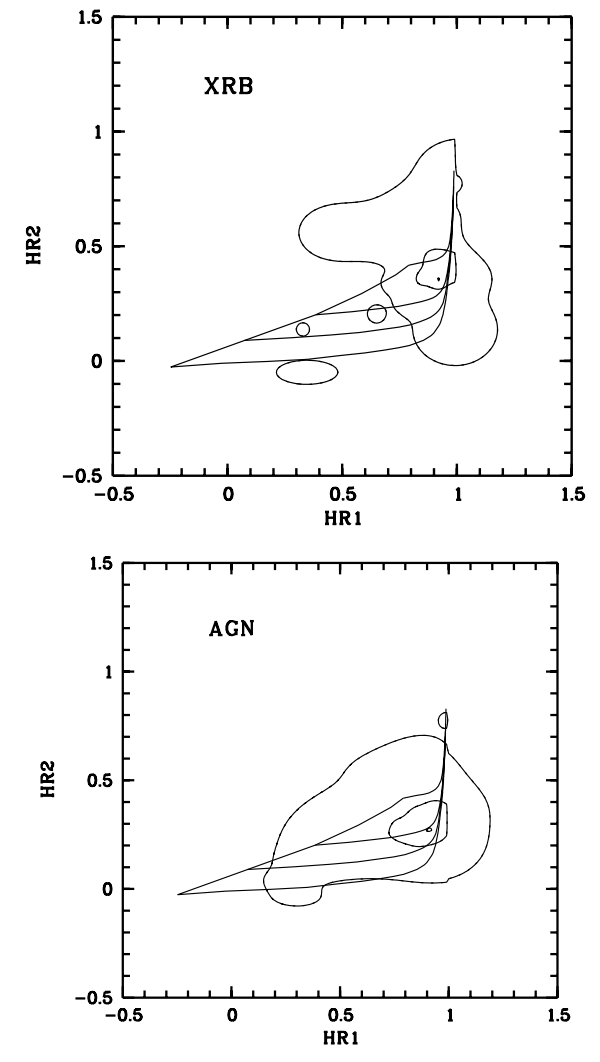

Fig. 4. Probability distribution of $H R 1$ and $H R 2$ values in the $H R 1$ - HR2 plane. The $68 \%, 95.4 \%$ and $99.7 \%$ confidence contours are given for sources with $\delta H R 1 \leq 0.2$ and $\delta H R 2 \leq 0.2$ from Table 1 classified as X-ray binaries (XRB, upper panel) and as AGN (lower panel). Also given are the tracks with constant powerlaw photon index $-\Gamma=1.0,2.0,2.5$ and 3.0.

From the location of a source in the $H R 1-H R 2$ plane a tentative source classification has been made. Sources which have hardness ratios which coincide with the range of $\Gamma$-tracks for X-ray binaries or AGN have been classified accordingly. In addition the galactic and LMC column at the location of a source has been used for a source classification. AGN are supposed to be seen through the galactic and total LMC absorbing column and X-ray binaries are seen through at least the galactic and at most the total LMC absorbing column. Of course there may be sources which have spectral properties which deviate from the standard values and the classification may not be unique. Especially the similarity between the spectral properties of the low-mass X-ray binary LMCX-2 and AGN is striking (LMCX-2 is located in the regime of AGN type spectra). A few of the sources which have not been classified as candidate X-ray binaries by HP99 and which show time variability in X-rays are located in the AGN regime and could also be time variable AGN. The source RX J0532.7-6926 (with number 914 in the catalog of HP99) has been classified as a LMXB by Haberl \& Pietsch (1999b) from a time variability analysis. From X-ray spectral fitting follows that this source has a very steep spectrum $(-\Gamma \sim 3.0)$ and could also be a time variable AGN. We did not include this source in the further analysis in the class of X-ray binaries.

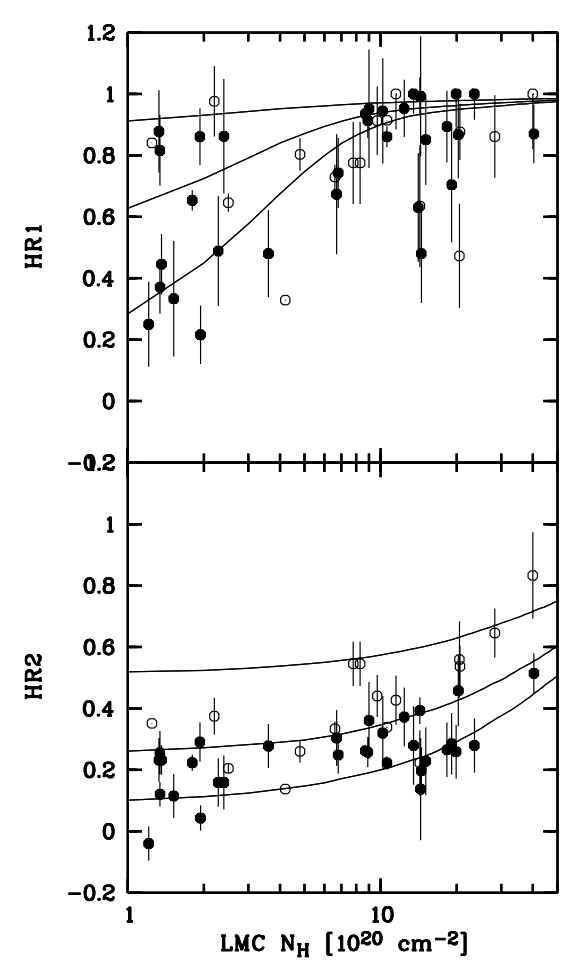

Fig. 5. Sources from Table 1 classified as background AGN or X-ray binaries and in the LMC $N_{\mathrm{H}}-H R 1$ and $H R 2$ plane. Tracks for three different powerlaw photon indices $-\Gamma=0.5$, 1.8 and 2.5 (upper to lower curves) are given. The sample of 49 AGN (filled circle) and X-ray binaries (open circle) is shown. Sources with accurate hardness ratio values $\delta H R 1 \leq$ 0.20 and $\delta H R 2 \leq 0.20$ have been used. The LMC $N_{\mathrm{H}}$ has been derived local to the X-ray source from 21-cm Parkes data and making use of the galactic foreground $N_{\mathrm{H}}$ derived from 21-cm Parkes data. Two AGN with $N_{\mathrm{H}}^{\mathrm{LMC}}<10^{20} \mathrm{~cm}^{-2}$ are not shown in the figure.

In the color - color diagram (HR1 - HR2 plane), Fig. 3, I show the location of the sources classified as X-ray binaries and as background AGN as given in Table 1. It is obvious that X-ray binaries and background AGN cover in general different areas in this diagram as expected due to the different steepness of their spectral slopes. Background AGN have steeper slopes and are found in regimes of lower values for HR2 than X-ray binaries (cf. Fig. 4) although there is some region of overlap (some X-ray binaries have as steep X-ray spectra as AGN). Due to absorption by galactic gas with column densities in the range $\sim(3-7) \times 10^{20} \mathrm{~cm}^{-2}$ the value of $H R 1$ does not extend to values $H R 1 \lesssim 0$.

I can obtain information about the total hydrogen column density due to LMC gas from the source shape. Due to the dependence of the ROSAT PSPC instrument point-spread function on the energy (the point-spreadfunction becomes narrower with increasing energy) AGN seen through high LMC columns appear sharp and pointlike while AGN seen through low LMC columns have broader images. This fact helps to confirm large LMC columns derived in the direction of background AGN. But some classified AGN may be X-ray galaxies and intrinsically extended so this argument is not a perfect one. 
A new source has been detected in the merged ROSAT $P S P C$ pointings (see Table 2) which is not contained in the catalog of HP99 ${ }^{1}$.

A second source is given which is not included in the catalog of HP99, the heavily absorbed background source RX J0532.0-6919 in the 30 Dor complex which coincides in position with the radio source MDM 65 of Marx et al. (1997). In addition two sources are given for which significantly improved positions (compared to those given in HP99) were derived.

Column 1 of the catalog of Table 2 gives the catalog index, Col. 2 the ROSAT source name, Cols. 3 and 4 the source position, the right ascension (RA) and declination (Dec) for the epoch J2000 with the 90\% confidence positional uncertainty (Col. 5), the likelihood of existence $L_{\mathrm{exi}}=-\ln (P)$ (Col. 6), with $P$ the probability that the detected source is due to excess counts measured above a mean local background. For the first source, RX J0529.4-6713, in addition to the coordinates the values of the hardness ratios $H R 1$ and $H R 2$ are given. The source parameters have been determined by applying the maximum likelihood source detection task to the merged data in the field of the corresponding source.

In Fig. $5 \mathrm{I}$ show the location of $49 \mathrm{X}$-ray sources from Table 1 with accurate values for the hardness ratios $\delta H R 1 \leq 0.20$ and $\delta H R 2 \leq 0.20$ and which have been classified either as background AGN or as X-ray binaries in the LMC $N_{\mathrm{H}}-H R 1$ and $H R 2$ plane respectively. The two AGN HP 37 and HP 352 which have $N_{\mathrm{H}}^{\mathrm{LMC}}<10^{20} \mathrm{~cm}^{-2}$ are not shown in this figure. The source HP 414 has not been included in the sample as it may be a foreground object and also the source HP 914 has not been included as it may either be a LMXB or an AGN. I also give tracks for powerlaw photon indices $-\Gamma=0.5,1.8$ and 2.5. Sources classified as XRB are preferrentially found in the $-\Gamma=0.5$ to 1.8 band while sources classified as AGN are preferentially found in the $-\Gamma=1.8$ to 2.5 band. There are a few exceptions, e.g. the XRB LMC X-4 has a steep powerlaw photon index and is outside the $-\Gamma=0.5$ to 1.8 band.

\footnotetext{
${ }^{1}$ It is a new supersoft source RX J0529.4-6713 at the southern boundary of the supergiant shell LMC 4. This source is very close to another source RX J0529.7-6713 which is contained in the HP99 catalog. Due to the closeness of both sources (the other source has been classified as an AGN) the source detection algorithm may have excluded this source. Spectral fitting using a blackbody spectral shape gives a temperature of $40 \mathrm{eV}$ and a luminosity of $\sim 2 \times 10^{36} \mathrm{erg} \mathrm{s}^{-1}$ if a galactic absorbing column density of $5.6 \times 10^{20} \mathrm{~cm}^{-2}$ and a LMC column density of $4.7 \times 10^{20} \mathrm{~cm}^{-2}$ is used. The spectrum is consistent with such absorption which is in favor for a supersoft source in the LMC, the southern region of LMC 4. The source appears to be variable in time. The large luminosity of the source would argue against a conventional $\mathrm{CV}$ nature of the source as for CVs luminosities do not exceed $\sim 10^{33} \mathrm{erg} \mathrm{s}^{-1}$. It thus could be a white dwarf with steady nuclear burning. Inside the $11^{\prime \prime}$ error circle no optical counterpart is seen in digital sky survey images (blue and red plates). There are 2 to 3 optical stellar objects at the periphery of the error circle.
}

Table 2. New X-ray sources detected in merged ROSAT PSPC observations and X-ray sources from the HP 99 catalog for which the positions have been improved.

\begin{tabular}{|c|c|c|c|c|c|}
\hline $\begin{array}{l}(1) \\
\text { Source } \\
\text { No. }\end{array}$ & $\begin{array}{c}(2) \\
\text { Source } \\
\text { Name } \\
\text { RX J }\end{array}$ & $\begin{array}{c}(3) \\
\text { RA } \\
(\mathrm{J} 2000) \\
\text { h m s }\end{array}$ & $\begin{array}{c}(4) \\
\text { Dec } \\
(\mathrm{J} 2000) \\
\circ,{ }^{\prime \prime}\end{array}$ & $\begin{array}{l}(5) \\
P_{\mathrm{e}} \\
\left({ }^{\prime \prime}\right)\end{array}$ & $\begin{array}{c}(6) \\
L_{\mathrm{ex}}\end{array}$ \\
\hline 1 & $0529.4-6713$ & 052925.8 & -671324 & 11 & 91 \\
\hline 2 & $0529.7-6713$ & 052947.0 & -671350 & 11 & 10 \\
\hline 3 & 0536.9-6913 & 053657.9 & -691329 & 17 & 125 \\
\hline 4 & $0553.2-7144$ & 055313.4 & -714403 & 25 & 11 \\
\hline
\end{tabular}

Notes on sources: Source 1: HR1 $=-0.849 \pm 0.142, H R 2=$ $0.474 \pm 0.020$, close to source 2; Source 2: HP 494, close to source 1; Source 3: $180 \mathrm{ksec}$ exposure, MDM65, see also Table 1; Source 4: HP 1303, $17^{\prime}$ off-axis.

The source classification has been made using the LMC $N_{\mathrm{H}}-H R 2$ diagram (in the LMC $N_{\mathrm{H}}-H R 1$ diagram there is more scatter as the value for $H R 1$ is less accurately determined than for $H R 2$ ).

\section{Constraining intervening $\mathrm{LMC}$ gas columns}

In Paper I I have determined the $N_{\mathrm{HI}}^{\mathrm{LMC}}$ values by performing X-ray spectral fitting for individual AGN. Here a different method, a hardness ratio analysis, has been chosen to constrain absorbing column densities. The chosen sample of AGN and candidate AGN comprises the AGN sample given in Paper I (22 AGN) and 64 additional candidate background X-ray sources. For $20 \mathrm{AGN}$ and candidate AGN values (with $1 \sigma$ errors) for the total LMC hydrogen absorbing column density $N_{\mathrm{H}}^{\mathrm{LMC}}$ could be derived and for additional 11 candidate AGN a range. For further 54 candidate AGN only $1 \sigma$ upper limits to the LMC gas column could be derived (and in addition in one case a $1 \sigma$ lower limit). It follows that the values derived for $N_{\mathrm{H}}^{\mathrm{LMC}}$ from the hardness ratio analysis are consistent with the values for the LMC absorbing column density derived from X-ray spectral fitting of Paper I.

I briefly outline the hardness ratio analysis method which has been applied. I simulated powerlaw tracks in the $H R 1$ - HR2 plane for a wide range of powerlaw photon indices $-\Gamma=(0.8-3.0)$. I compared the location of the hardness ratio error ellipses of individual AGN and candidate AGN with respect to these tracks to infer the absorbing column densities of LMC gas in the direction of individual background X-ray sources. I assumed that the powerlaw photon indices of background sources are in the range $-\Gamma=(2.0-2.5)$, which is the range of powerlaw photon indices derived by Brinkmann et al. (2000) from the X-ray spectra of a large sample of AGN. In the simulations reduced metallicities $(-0.3$ dex relative to galactic interstellar absorption abundances) have been assumed for the LMC gas and galactic interstellar abundances for the galactic foreground gas. In Table 1 the values for the galactic and LMC hydrogen column density $N_{\mathrm{HI}}^{\mathrm{gal}}$ and $N_{\mathrm{HI}}^{\mathrm{LMC}}$ are 


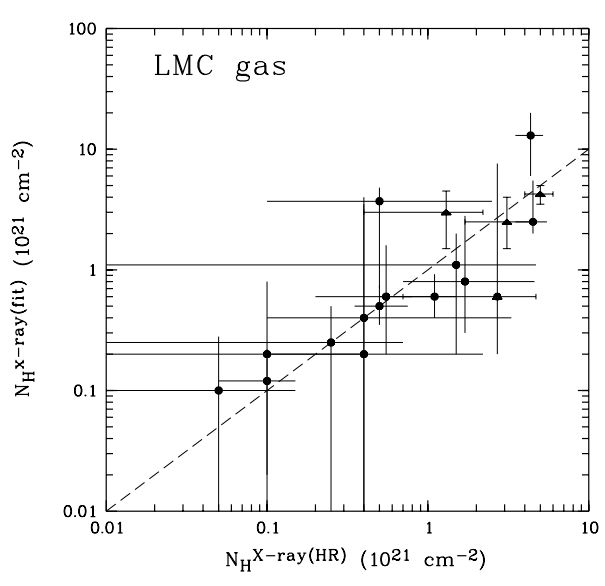

Fig. 6. Correlation between LMC hydrogen absorbing column density (after galactic foreground gas has been removed) as derived from the hardness ratio analysis compared with the LMC hydrogen absorbing column density derived from the $\mathrm{X}$-ray spectral fit (cf. Paper I). The dashed line gives the linear relation for which both LMC column density determinations are equal.

given which have been derived from 21-cm H I surveys of the LMC field performed with the Parkes radio telescope (Brüns et al. 2001, see also Dickey \& Lockman 1990). Gas columns derived from 21-cm measurements can be separated into a galactic and a LMC component due to the different systemic velocities of both components. In addition the total LMC absorbing hydrogen column density $N_{\mathrm{H}}^{\mathrm{LMC}}$ derived from the hardness ratio analysis is given.

In Fig. 6 I show the correlation between the LMC hydrogen absorbing column density derived with the hardness ratio analysis in comparison with the LMC hydrogen absorbing column density derived from the X-ray spectral fit (Paper I). There is a linear correlation between the LMC gas columns determined by both methods which gives reliability to the LMC gas columns derived by both methods.

I present in Fig. 7 the comparison between the LMC gas columns inferred from the 21-cm H I Parkes survey and the LMC gas columns inferred from the hardness ratio analysis for background sources in addition to those for which X-ray spectral fitting has been performed in Paper I (for 6 AGN a best-fit for the LMC $N_{\mathrm{H}}$ value, for 7 AGN a range of values, for 50 AGN upper limits and for one AGN a lower limit is given). The values for the LMC gas columns inferred from the hardness ratio analysis agree in most cases within the uncertainties with the LMC H I columns inferred from the Parkes survey.

\subsection{Deriving constraints on the LMC metallicity}

It is assumed that AGN have canonical powerlaw photon indices $-\Gamma=2.0$ to 2.5 in the ROSAT PSPC band. From simulations, tracks for constant powerlaw indices $-\Gamma$ and a galactic foreground absorbing column of $3 \times 10^{20} \mathrm{~cm}^{-2}$ have been derived in the $H R 1-H R 2$ plane by varying the LMC absorbing column density $N_{\mathrm{H}}$.
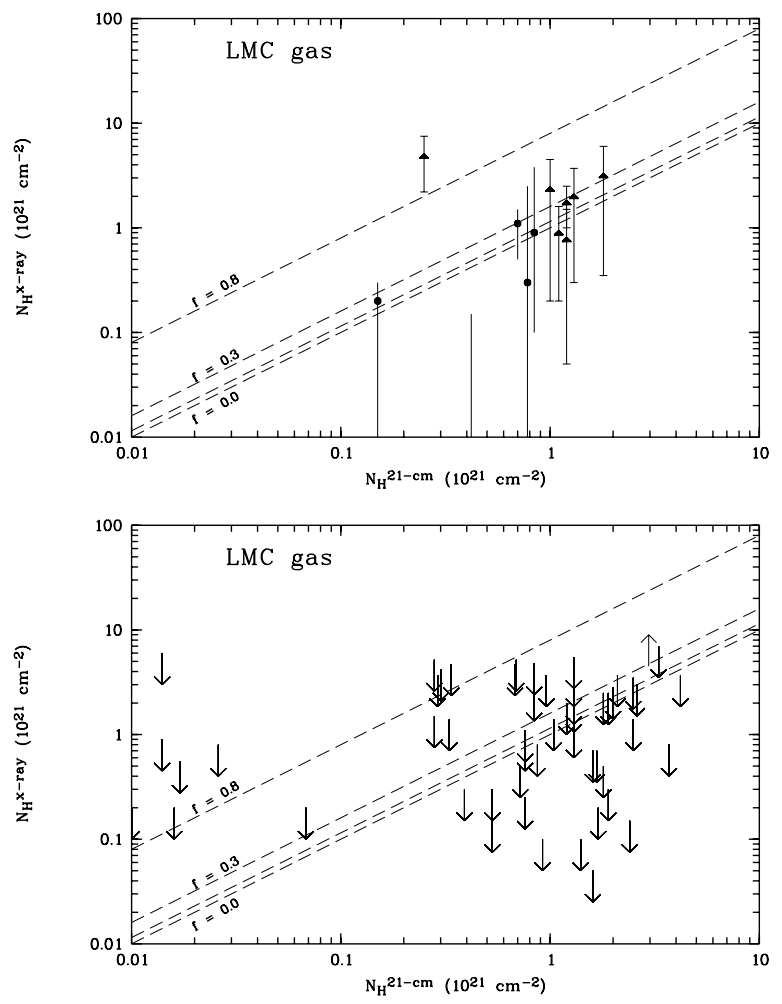

Fig. 7. LMC hydrogen absorbing column density (after galactic foreground gas has been removed) as derived from the hardness ratio analysis assuming constraints on the powerlaw photon index (cf. Table 1). Upper panel: AGN (additional to the sample from Paper I) for which a best-fit has been determined (the best-fit value is given as filled circle and $1 \sigma$ error bars are drawn) and AGN for which an $N_{\mathrm{H}}$ range, band, has been determined and for which the mean value is shown as a filled triangle and $1 \sigma$ error bars are drawn with small cross bars. Lower panel: AGN for which only upper limits (and in one case a lower limit) to the LMC gas columns has been determined. With dashed lines the dependences on the molecular mass fraction $f=0,0.1,0.3$ and 0.8 are indicated.

For the LMC gas hybrid models have been used assuming a constant foreground hydrogen column due to the Milky Way gas of $3 \times 10^{20} \mathrm{~cm}^{-2}$. In the simulations the metallicity has been varied from -0.8 dex to +0.5 dex in steps of 0.05 dex. In addition $-\Gamma$ has been varied from 2.1 to 2.4 for the AGN and from 1.4 to 1.7 for the X-ray binaries. Three AGN (HP 54, HP 380 and HP 1094, cf. Paper I) with accurately determined values for the hardness ratios $H R 1$ and $H R 2$ could be used to constrain the metallicity. From the location of these AGN in the HR1 - HR2 plane metallicities somewhat in excess of galactic metallicities $X>0.1$ can be excluded. Metallicities as low as $X=-0.7$ were found to be consistent with the data.

The size of the sample has been extended in a next step and the mean metallicity of the intervening LMC gas and the powerlaw slope of the flux have been determined in a least-square grid search. This search has been performed for two different samples, an AGN sample with 14 objects and an X-ray binary sample with 9 objects (cf. Table 1). 

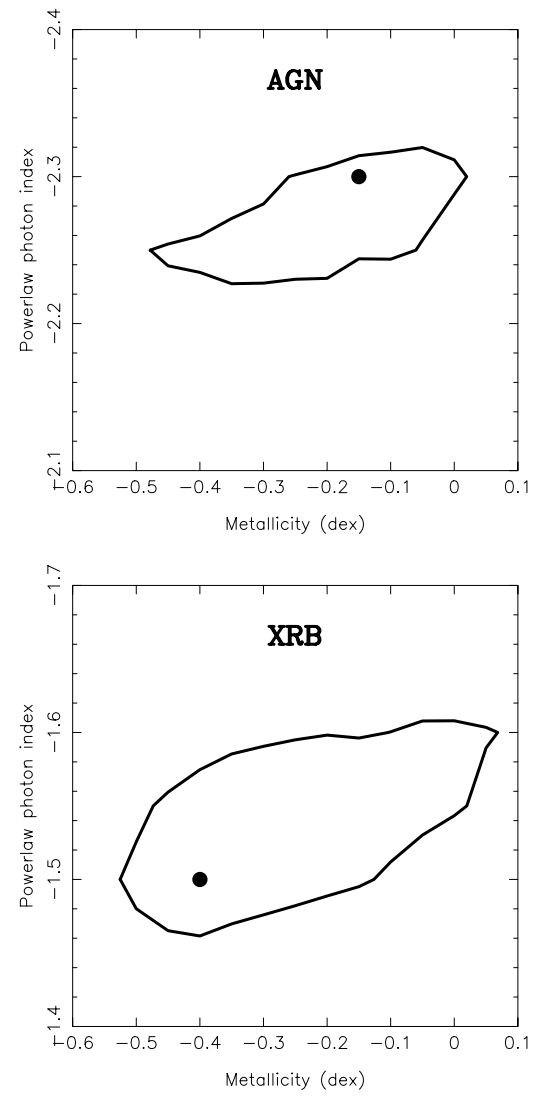

Fig. 8. Upper panel: confidence contours (99\%) for 14 candidate AGN with catalog number 1, 37, 54, 101, 147, 380, 411, 561, 653, 876, 1040, 1094, 1181, and 1247 (Haberl \& Pietsch 1999) and hardness ratio errors $\delta H R<0.20)$ in the $\Gamma-X$ plane. Lower panel: confidence contours for $9 \mathrm{X}$-ray binaries (XRB) with catalog number 41, 106, 184, 204, 252, 436, 1001, 1225 , and 1325 and $\delta H R<0.20$ in the $\Gamma-X$ plane.

These objects were taken from a sample selected in this work and, in addition, only objects with accurate hardness ratios $\delta H R<0.20$ were used.

From the formal fit it is found that the powerlaw slope $\Gamma$ and the metallicity $X$ can be constrained for both samples (the AGN and the X-ray binary sample). In the case of the AGN, the errors in the hardness ratios of the AGN have been increased by a factor of 1.4. The range of metallicities which is derived in this way is in agreement with the range of tracks for different metallicities which is covered by the used data points. In the case of the X-ray binaries, a systematic offset of 0.03 in the values of the hardness ratios has been assumed in the fit. This avoids that the least-square fit is biased towards the data points with very small error bars in the hardness ratio values as derived for the bright X-ray binaries (e.g. LMC X-1). This has the effect of increasing the parameter range for a given confidence (e.g. the $99 \%$ confidence which is shown in Fig. 8).

In Fig. 8 the confidence contours are shown for the $14 \mathrm{AGN}$ and the $9 \mathrm{X}$-ray binaries in the HR1 - HR2 plane. It is found that for the X-ray binaries the powerlaw slope can be confined to $-\Gamma=1.45$ to 1.6 and the metallicity to $X=-0.5$ to +0.0 (99\% confidence). For the 14 AGN (Fig. 8) I find that the powerlaw slope can be confined to $-\Gamma=2.2$ to 2.3 and the metallicity to $X=-0.6$ to +0.15 (99\% confidence). The best-fit metallicity is $-X=0.4$ for $\mathrm{X}$-ray binaries and $X=-0.15$ for AGN respectively.

The value for the metallicity which has been found from the AGN and the X-ray binary sample is consistent with the metallicity of -0.2 to -0.6 derived for the LMC (cf. Pagel 1993; Russell \& Dopita 1992). The powerlaw photon index derived for the AGN sample of $-\Gamma=2.2$ to 2.3 is consistent with the powerlaw slope derived for AGN type spectra (cf. discussion in Paper I). The powerlaw photon index derived for the X-ray binaries $-\Gamma=1.45$ to 1.6 is steeper than the canonical value of $-\Gamma \sim 1.0$ (see also Sect.6). Apparently for the LMC X-ray binaries steeper powerlaw photon indices are observed in the ROSAT PSPC band. From spectral fitting applied to bright LMC X-ray binaries (LMC X-1, LMC X-2, LMC X-3 and LMC X-4) follows that simple powerlaw spectra cannot explain the absorbed spectra and more complicated spectral shapes have to be fitted.

\section{The $X$-ray binary sample}

For the X-ray binary sample all classified X-ray binaries given in HP99 have been considered. Three of these X-ray binaries were found to be slightly outside the hardness ratio selection criteria but were also included in the sample. In addition sources were taken into account which were observed in the central $20^{\prime}$ of the detector, which fulfilled the selection criteria for X-ray binaries given in Sect. 2, i.e. sources which were located in the $H R 1-H R 2$ plane "above" the AGN band (cf. Sect. 4). In addition an X-ray spectral fit has been applied to the spectra of these sources and the consistency with an X-ray binary has been checked. Also a time variability study of the source count rate has been performed. There were 30 sources found which were classified as (candidate) X-ray binaries (cf. Table 1). 15 of these sources have more than 50 observed counts and powerlaw photon indices were derived for these sources (excluding HP 914). It is found that the distribution of powerlaw photon indices is consistent with a mean $\Gamma$ of -1.4 and a $\sigma$ of 0.9 . But $\Gamma$ strongly depends on the used value of the galactic and LMC absorbing column. For most of the X-ray binaries the total LMC columns have been used in the spectral fit. This assumption need not always be correct and I have taken this fact into account in a few cases where the $N_{\mathrm{H}}$ value could be determined in the spectral fit.

If one compares the number of classified AGN and X-ray binaries it is found that a fraction of $80 \%$ of the spectrally hard X-ray sources with more than 50 detected counts are AGN and 20\% are X-ray binaries.

For the 30 (candidate) X-ray binaries the unabsorbed flux and the luminosity $(0.1-2.4) \mathrm{keV}$ have been determined in an X-ray spectral fit. The derived flux and luminosity histograms are given in Fig. 9. It follows that 


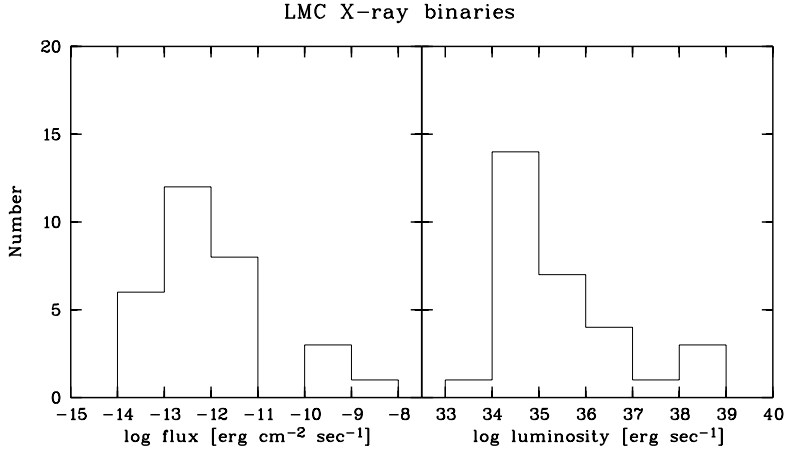

Fig. 9. Distribution of flux and luminosity corrected for absorption (left and right panel respectively) for the 30 (candidate) X-ray binaries in the observed field of the LMC (cf. Table 1). The number of X-ray binaries per flux and luminosity bin is given.

there are $3,4,8,15$, and $29 \mathrm{X}$-ray binaries with luminosities in excess of $10^{38}, 10^{37}, 10^{36}, 10^{35}$, and $10^{34} \mathrm{erg} \mathrm{s}^{-1}$ respectively.

These numbers can be compared with the number of X-ray binaries predicted from stellar evolutionary calculations for the LMC (Dalton \& Sarazin 1995). According to these calculations there are $1,5,18,125$, and 750 X-ray binaries predicted to exist in the LMC with luminosities in excess of $10^{38}, 10^{37}, 10^{36}, 10^{35}$, and $10^{34} \mathrm{erg} \mathrm{s}^{-1}$ respectively. Such a comparison will only be valid if the sample of X-ray binaries selected here is complete. There are two factors which have to be taken into account for such a completeness consideration, the sensitivity limit of the LMC $\mathrm{X}$-ray survey and the fraction of the LMC disk covered by the observations.

In Paper III it will be shown that our survey is complete in the observed field to a flux of $\sim 10^{-12} \mathrm{erg} \mathrm{cm}^{-2} \mathrm{~s}^{-1}$ which is equivalent to a luminosity of $3 \times 10^{35} \mathrm{erg} \mathrm{s}^{-1}$. Our observations cover 16 square degrees, which is $\sim 24 \%$ of the LMC disk. Assuming that X-ray binaries are homogenously distributed across the LMC disk, we extrapolate from the number of 8 observed X-ray binaries with luminosities in excess of $10^{36} \mathrm{erg} \mathrm{s}^{-1}$ that there may be 33 X-ray binaries across the whole LMC disk above this luminosity limit. If one compares this with the number of 18 X-ray binaries predicted from population synthesis calculations then an excess of X-ray binaries appears to exist. But a detailed investigation of the candidate X-ray binaries is required to give reliability to a deviation in these numbers. For a flux in excess of $3 \times 10^{-13} \mathrm{erg} \mathrm{cm}^{-2} \mathrm{~s}^{-1}$ (which corresponds to a luminosity of $10^{35} \mathrm{erg} \mathrm{s}^{-1}$ ) 15 X-ray binaries are in our observed sample. Assuming besides the incompleteness due to the covered LMC field the incompleteness due to the given sensitivity (which is about a factor of 1.3, cf. Paper III) we derive an extrapolated population of $81 \mathrm{X}$-ray binaries. This population would be less than the predicted $125 \mathrm{X}$-ray binaries but the extrapolated number my have large uncertainties and such a comparison may not be too reliable.

\subsection{Comparison with the number of $X$-ray binaries in the SMC}

Two of the newly classified X-ray binaries, RX J0523.27004 and RX J0527.1-7005, are located in the optical bar of the LMC (cf. Table 1). Another source newly classified as an X-ray binary, RX J0524.2-6620, lies in the eastern H I shell of the supergiant shell LMC 4. An additional source which is contained in Table 1 in the section of background AGN, RX J00546.8-6851, but which may be an X-ray binary (see also Paper I and Sasaki et al. 2000) is located in or at least very close to the supergiant shell LMC 2. In total, 9 of the 30 sources classified as X-ray binaries (i.e. $30 \%$ ) are associated with the supergiant shell LMC 4. This could be a selection effect as the LMC 4 region has been observed during many ROSAT pointings. But also other regions of the LMC, e.g. the 30 Dor area, have been observed during multiple observations and less X-ray binaries have been detected in these areas. Assuming that these sources are high-mass X-ray binary systems which have formed within an evolutionary time scale of $\sim 10^{7}$ years (cf. Popov et al. 1998) may indicate that star formation has taken place in the last 10 million years in the LMC disk (including the $\mathrm{H}$ I boundary of the supergiant shell LMC 4). To find candidate high-mass X-ray binaries in the LMC may be of importance as recent X-ray surveys of the Small Magellanic Cloud (SMC) have revealed a large number of such systems showing X-ray pulsations in this other Magellanic Clouds galaxy (cf. Yokogawa et al. 2000; Finger et al. 2001). One scenario put forward to explain the large number of high-mass X-ray binaries discovered in the SMC is the trigger of star formation during the recent close encounter between the SMC and the LMC $\sim(0.2-0.4)$ Gyr ago (cf. Gardiner et al. (1994, hereafter GSF94); Gardiner \& Noguchi (1996, hereafter GN96)). In such a scenario it is expected that star formation was also triggered in the LMC (cf. van den Bergh 2000, for a recent update of the star formation rate of the LMC during the last 9 Gyr). Finding new candidate high-mass X-ray binaries in the LMC which are associated with at least two supergiant shells may be consistent with such a scenario.

Can this scenario account for the observed number of candidate high-mass X-ray binaries in the LMC and SMC. In the previous section we estimated an extrapolated number of $33 \mathrm{X}$-ray binaries with luminosities in excess of $10^{36} \mathrm{erg} \mathrm{s}^{-1}$ in the LMC field. A comparable number for the population of high-mass X-ray binaries in the SMC has been set up by Haberl \& Sasaki (2000) who recently increased the number of detected Be-type X-ray binaries in the SMC to 50. Assuming that at least $40 \%$ of these X-ray binaries have outburst luminosities in excess of $10^{36} \mathrm{erg} \mathrm{s}^{-1}$ would give a ratio of LMC to SMC high-mass X-ray binaries of $\sim(0.7-1.7)$. An additional uncertainty in these numbers may be due to the fact that not all Be-type X-ray binaries have so far been detected in the LMC and the SMC (either in quiescence or in outburst). A value for the number ratio of $\sim(0.7-1.7)$ is not in agreement with the mass ratio of both galaxies of $\sim 10$ 
(the mass of the LMC and the SMC is $\sim 2 \times 10^{10} M_{\odot}$ and $\sim 2 \times 10^{9} M_{\odot}$ respectively, cf. GSF94). It would be more consistent with the ratio of the gas mass of both galaxies of $\sim(1.2-1.8)$ (the H I mass of the LMC and SMC is $\sim 5.2 \times 10^{8} M_{\odot}$ (Kim et al. 1998) and $\sim 4.2 \times 10^{8} M_{\odot}$ (Stanimirovic et al. 1999) respectively, and for the LMC the gas mass may be larger than the $\mathrm{H}$ I mass by $\sim 40 \%$ due to the contribution of molecular hydrogen). Assuming that the star formation rate is proportional to the gas mass of a galaxy, the comparable gas mass of the SMC and the LMC may give an explanation for the comparable number of high-mass X-ray binaries found in both galaxies.

Star formation may have been triggered during an encounter of these two galaxies. Assuming that during the encounter turbulence was introduced into the gaseous phase of the galaxy disk, from the condition of conservation of angular momentum constraints can be derived for the ratio of star formation rates $S F R$ induced in both galaxies. Making use of the formalism for the star formation rate given by Kennicutt (1998) in which the star formation rate scales with the gas density and the orbital time scale and which has been found to give a good fit for a large sample of normal and starburst galaxies, then one finds that this ratio can be expressed ${ }^{2}$ as

$$
\frac{S F R_{\mathrm{LMC}}}{S F R_{\mathrm{SMC}}} \approx\left(\frac{R_{\mathrm{SMC}}}{R_{\mathrm{LMC}}}\right)^{2}
$$

with $R$ the radius of the gaseous disk of a galaxy. If one uses for the LMC $R_{\mathrm{LMC}}=3.7 \mathrm{kpc}$ (Kim et al. 1998) and for the SMC $R_{\mathrm{SMC}}=2.3 \mathrm{kpc}$ (e.g. Stanimirovic et al. $1999)$, then one obtains $\frac{S F R_{\mathrm{LMC}}}{S F R_{\mathrm{SMC}}} \approx 0.4$.

If one assumes that the starburst was efficient enough to significantly increase the star formation rate preferentially in the SMC and that the number of high-mass X-ray binaries scales with the star formation rate of a galaxy at an epoch of $\sim 10^{7}$ years ago (which may be somewhat earlier if a delay for the onset of star formation is taken into account) then one can directly compare the ratio of

\footnotetext{
${ }^{2}$ Equation (3) has been derived assuming conservation of angular momentum during the encounter of the LMC and the SMC. As a result the SMC disk was spun up to a higher angular velocity than the LMC disk (but see Maragoudaki et al. 2001). This resulted in a more efficient star formation burst in the SMC compared to the LMC. The history of the interaction, i.e. the state of the system prior to the encounter, and the distance of both galaxies during the encounter do not enter into this consideration. Only the ratio of the star formation rates induced by the encounter of both galaxies is given. Only under the assumption that the encounter was close enough to enhance the star formation rate efficiently Eq. (3) may be of relevance for the consideration of the total star formation rate during the encounter. Different values have been derived from $N$-body and SPH simulations of such a galaxy-galaxy encounter for the distance of closest approach, $7 \mathrm{kpc}$ (GSF94, GN96), and $20 \mathrm{kpc}$ (Li \& Thronson 1999), respectively. Any determination of the absolute value of the induced star formation rate depends on the specific assumptions made about the history of this galaxy encounter.
}

the star formation rates of two galaxies during this epoch with the ratio of presently observed numbers of high-mass $\mathrm{X}$-ray binaries in these galaxies. The ratio of high-mass $\mathrm{X}$-ray binaries in the LMC to those in the SMC is derived from the observed numbers to be $\sim(0.7-1.7)$. There appear to be many more X-ray binaries in the LMC than predicted from Eq. (3). One explanation may be that the formation of high-mass X-ray binaries in the LMC is less affected by the starburst than in the SMC, i.e. in the LMC we observe the constant star formation with a minor contribution from a starburst.

From the OGLE survey of 93 star clusters in a field in the central $2.4 \mathrm{deg}^{2}$ of the SMC Pietrzynski \& Udalski (1999) derived that most of these star clusters are younger than $\sim 20 \times 10^{7}$ years. This finding could mean that the formation of star clusters during the last $(20-30) \times 10^{7}$ years was enhanced at least in the central field of the LMC. Alternatively it may be explained by an efficient process of disintegration of clusters older than $(20-30) \times 10^{7}$ years. Both effects may be explained by a tidal interaction of the SMC with the LMC which may have resulted in a burst of cluster formation and/or in the disruption of pre-existing stellar clusters.

\section{Summary and conclusions}

The sample of spectrally hard X-ray sources in the field of the LMC observed with the ROSAT PSPC and published in the catalog of HP99 has been reinvestigated. Especially accurate values for the count rate have been determined in the spectrally hard $(0.5-2.0 \mathrm{keV})$ and broad $(0.1-2.4 \mathrm{keV})$ band respectively and values for the hardness ratios $H R 1$ and HR2 have been determined making use of merged data in the direction of each investigated X-ray source. The analysis has been restricted to X-ray sources which have been observed in the inner $20^{\prime}$ of the PSPC detector.

Simulations have been performed to derive tracks for powerlaw spectra with slopes comprised by X-ray binaries and AGN in the HR1 - HR2 plane. In these simulations a wide range of metallicities for the LMC gas has been considered. Comparing the location of the X-ray sources in the $H R 1$ - HR2 plane with respect to the simulated tracks for X-ray binaries and AGN a source classification has been achieved of the sample of spectrally hard X-ray sources observed in the central $20^{\prime}$ of the PSPC detector. 141 sources have been classified as AGN (or as likely AGN) and 30 sources as X-ray binaries (or as likely XRB). This means that $82 \%$ of the classified hard X-ray sources in the LMC field are AGN and $18 \%$ are X-ray binaries.

I constrained, for 31 of these AGN (18 in addition to sources already investigated in Paper I), the LMC gas columns from the location of these sources in the HR1 $H R 2$ plane. In addition I derived for 54 AGN upper limits for the LMC gas columns.

I independently constrained the metallicity of the LMC gas by fitting simulated tracks of constant powerlaw slopes in the $H R 1$ - HR2 plane to the observation derived $H R 1$ and $H R 2$ values for the AGN and the X-ray binary sample. 
I found that the required metallicity of the LMC gas is in the range -0.6 to +0.1 dex at $99 \%$ confidence.

$\mathrm{I}$ also established the catalog of X-ray sources in a deep merged observation of the field of the supergiant shell LMC 4. I detected 97 X-ray sources of which I classified 35 sources as candidate AGN.

The number of 30 (candidate) X-ray binaries observed in the LMC is compared with the number of X-ray binaries predicted from population synthesis calculations for the LMC. In addition the number of (candidate) highmass X-ray binaries observed in the LMC with luminosities in excess of $10^{36} \mathrm{erg} \mathrm{s}^{-1}$ is compared with the number of high-mass X-ray binaries in the SMC. It is found that the comparable number of high-mass X-ray binaries scale with the comparable gas mass of the host galaxies. The number of high-mass X-ray binaries may have been preferentially enhanced in the SMC due to a star formation burst initiated by the LMC-SMC galaxy encounter.

\section{Appendix A: $X$-ray sources in a deep merged observation of the northern field of the supergiant shell LMC 4}

In the previous analysis in this paper I have made use of the X-ray sources given in the catalog of HP99. Part of the LMC region has been observed during several observations and a large integrated exposure exists for these fields. In particular I investigated the field of the northern area of the supergiant shell LMC 4 to find out how many X-ray sources can be detected in deep merged observations of this specific field.

LMC 4 is the northern and largest (with a diameter of $\sim 1200 \mathrm{pc}$ ) of five supergiant shells in the LMC which are characterized by circular regions of filamentary H II emission (Meaburn 1980). McGee \& Milton (1966) noted the existence of a deep minimum in the column density of neutral hydrogen in LMC 4 in their 21-cm H I observations. Inside this hole is the stellar association Shapley III (Nail \& Shapley 1953).

I merged the observations ${ }^{3}$ existing for the field in the northern area of the supergiant shell LMC 4 making use of source detection routines (local, map, and maximum likelihood) which are available in EXSAS. I applied the local, map, and the maximum likelihood source detection task to one energy band $(0.5-2.4 \mathrm{keV})$ to derive the catalog of sources. $97 \mathrm{X}$-ray sources were detected in the 1.8 square degree field. I accepted only sources with a likelihood ratio of existence $>8$ and checked the reality of the sources on a displayed image. The catalog of these sources is given in Table 3. Column 1 gives the source index, Col. 2 the ROSAT source name, Cols. 3 and 4 the source position, the right ascension (RA) and the declination (Dec) for the epoch J2000 with the $90 \%$ confidence positional uncertainty (Col. 5), the count rate $(0.1-2.4 \mathrm{keV}$, Col. 6$)$, the hardness ratios HR1 and HR2 (Col. 7 and 8), the source

\footnotetext{
3 The center of the merged observation is RA $05^{\mathrm{h}} 29^{\mathrm{m}} 00^{\mathrm{s}}$,
} Dec $-66^{\circ} 03^{\prime} 04^{\prime \prime}$. extent in arcsec in case the extent likelihood ratio is $>10$ (Col. 9), the likelihood ratio of existence $L_{\text {exi }}=-\ln (P)$, with $P$ the probability that the detected source is due to excess counts measured above a mean local background (Col. 10), the distance of the source from the center of the field of the merged observation (Col. 11), the source index from the catalog of HP99 (Col. 12), the distance to that source in arcsec (Col. 13), the classification of the source (with $\mathrm{A}=\mathrm{AGN}, \mathrm{B}=\mathrm{X}$-ray binary, $\mathrm{R}=$ supernova remnant, $\mathrm{F}=$ foreground star) in Col. 14 and remarks in Col. 15 .

The exposure time of the merged observation varies over a large range and has a region of high exposure ( $\sim 50$ to $70 \mathrm{ksec}$ ) in one merged PSPC pointing. The limiting flux for the sources detected in this merged observation and given in the catalog of Table 3 is $8 \times$ $10^{-15} \mathrm{erg} \mathrm{cm}^{-2} \mathrm{~s}^{-1}$ assuming a powerlaw spectrum with a photon index of $-\Gamma=2$ and a galactic absorbing column density of $5 \times 10^{20} \mathrm{~cm}^{-2}$.

If one considers the location of the sources detected in this field with accurate values for the hardness ratios $\delta H R 1 \leq 0.20$ and $\delta H R 2 \leq 0.20$ in the hardness ratio $H R 1$ - HR2 plane then one finds that most of the sources are located in the region bounded by the powerlaw tracks $-\Gamma=1.0,2.0$ and 3.0 and are consistent with AGN and X-ray binaries (cf. Sect. 4). A few bright sources which are located outside this region are (consistent with) supernova remnants.

A fraction of the detected sources is contained in the sample of sources investigated in the previous sections and is also given in Table 1. 60 sources correlate within a search radius of $20^{\prime \prime}$ with a source in the catalog of Haberl \& Pietsch (1999). I made a tentative classification of the $97 \mathrm{X}$-ray sources detected in the field of the supergiant shell LMC 4 from the location of these sources in the HR1 - HR2 plane (cf. Sect. 4.1). I classified 35 of the sources with more than 30 observed counts as AGN (or likely AGN). This low threshold in counts has been chosen as the X-ray survey in the field of the Supergiant Shell LMC 4 was considerably deeper than the X-ray survey of the general LMC field. In Paper III it will be made use of this AGN sample to derive the $\log N-\log S$ of background X-ray sources in the field of the Supergiant Shell LMC 4.

Acknowledgements. The ROSAT project is supported by the Max-Planck-Gesellschaft and the Bundesministerium für Forschung und Technologie (BMFT). This research has made use of the SIMBAD data base operated at CDS, Strasbourg, France. I thank C. Brüns for making available the Parkes 21-cm map of the galactic and LMC HI in the field of the LMC. I thank J. Kerp for comments on an earlier version of the manuscript. I thank K. S. de Boer for suggestions to improve the article. I thank an anonymous referee for the suggestions to improve the manuscript. PK is supported by the Graduiertenkolleg on the "Magellanic Clouds and other Dwarf galaxies" (DFG GRK 118). 


\section{References}

Brinkmann, W., Yuan, W., \& Siebert, J. 1997, A\&A, 319, 413 Brinkmann, W., Laurent-Muehleisen, S. A., Voges, W., et al. 2000, A\&A, 356, 445

Brüns, C., Kerp, J., Staveley-Smith, L. 2001, in Mapping the Hidden Universe: The Universe Behind the Milky Way The Universe in HI, ed. R. C. Kraan Korteweg, \& P. A. Henning, ASP Conf., 218, 349

Chu, Y.-H., Kim, S., Points, S. A., et al. 2000, AJ, 119, 2242

Coe, M. J., Negueruela, I., Buckley, D. A. H., et al. 2001, MNRAS, 324, 623

Comastri, A., Setti, G., Zamorani, G., \& Hasinger, G. 1995, A\&A, 296, 1

Cowley, A. P., Schmidtke, P. C., McGrath, T. K., et al. 1997, PASP, 109, 21

Cusumano, G., Israel, G. L, Mannucci, F., et al. 1998, A\&A, 337,772

Dalton, W. W., Sarazin, C. L. 1995, High-Mass X-ray Binary Modeling: The Magellanic Clous Populations (preprint)

Dickey, J. M., \& Lockman, F. J. 1990, ARA\&A, 28, 215

Filipovic, M. D., Haynes, R. F., White, G. L., et al. 1995, A\&AS, 111, 311

Filipovic, M. D., Haynes, R. F., White, G. L., \& Jones, P. A. 1998, A\&AS, 130, 421

Finger, M. H., Macomb, D. J., Lamb, D. C., et al. 2000, ApJ, 560,378

Gardiner, L. T., Sawa, T., \& Fujimoto, M. 1994, MNRAS, 266, 567 [GSF94]

Gardiner, L. T., \& Noguchi, M. 1996, MNRAS, 278, 191 [GN96]

Gilli, R., Risaliti, G., \& Salvati, M. 1999, A\&A, 347, 424 [GRS99]

Gilli, R., Salvati, M., \& Hasinger, G. 2001, A\&A, 366, 407

Gochermann, J., Grothues, H.-G., Österreicher, M. O., et al. 1993, A\&AS, 99, 591

Grothues, H.-G., Österreicher, M. O., Gochermann, I., et al. 1997, A\&AS, 121, 247

Haberl, F., Pietsch, W., \& Dennerl, K. 1995, A\&A, 303, L49

Haberl, F., \& Pietsch, W. 1999a, A\&A, 139, 277 [HP99a]

Haberl, F., \& Pietsch, W. 1999b, A\&A, 344, 521 [HP99b]

Haberl, F., Filipovic, M. D., Pietsch, W., \& Kahabka, P. 2000, A\&AS, 142, 41

Haberl, F., \& Sasaki, M. 2000, A\&A, 359, 573

Haberl, F., \& Pietsch, W. 2001, A\&A, 373, 438

Hasinger, G., Burg, R., Giacconi, R., et al. 1993, A\&A, 275, 1

Hasinger, G., Burg, R., Giacconi, R., et al. 1998, A\&A, 329, 482

Kahabka, P., de Boer, K. S., \& Brüns, C. 2001, A\&A, 371, 816 (Paper I)

Kahabka, P., de Boer, K. S., \& Brüns, C. 2002, A\&A, 388, 113 (Paper III)

Kennicutt, R. C. 1998, ApJ, 498, 541

Kim, S., Staveley-Smith, L., Dopita, M. A., et al. 1998, ApJ, 503,674

Kim, S., Dopita, M. A., Staveley-Smith, \& Bessell, M. S. 1999, AJ, 118, 2798

Kontizas, M., Morgan, D. H., Hatzidimitrou, D., \& Kontizas, E. 1990, A\&AS, 84, 527

Li, P. S., \& Thronson, H. A. 1999, in New Views of the Magellanic Clouds, ed. Y.-H. Chu, N. B. Suntzeff, J. E. Hesser, \& D. A. Bohlender, IAU Symp., 190, 503

Liu, Q. Z., van Paradijs, J., \& van den Heuvel, E. P. J. 2000, A\&AS, 147, 25
Maragoudaki, F., Kontzias, M., Morgan, D. H., et al. 2001, A\&A, 379, 864

Massey, P., Waterhouse, E., DeGioia-Eastwood, K. 2000, AJ, 119,221

Marx, M., Dickey, J. M., \& Mebold, U. 1997, A\&AS, 126, 325

McGee, R. X., \& Milton, J. A. 1966, Aust. J. Phys., 19, 343

Meaburn, J. 1980, MNRAS, 192, 365

Morrison, R., \& McCammon, D. 1983, ApJ, 270, 119

Nail, V. McK., Shapley, H. 1953, Proc. Nat. Acad. Sci. USA, 39,358

Olsen, K. A. G., Hodge, P. W., Wilcotes, E. M., \& Pastwick, L. 1997, ApJ, 475, 545

Pagel, B. E. J. 1993, Stellar vs. Interstellar Abundances in the Magellanic Clouds, in Lecture Notes in Physics 416, New Aspects of Magellanic Cloud Research, ed. B. Baschek, G. Klare, \& J. Lequeux, 330

Pietrzynski, G., \& Udalski, A. 1999, Acta Astron., 49, 157

Pietsch, W., \& Kahabka, P. 1993, ROSAT Observations of the Magellanic Clouds, in New Aspects of Magellanic Cloud Research, Lecture Notes in Physics 416, ed. B. Baschek, G. Klare, \& J. Lequeux, 59

Popov, S. B., Lipunov, V. M., Prokhorov, M. E., et al. 1998, Astron. Reports, 42, 29

Rebeirot, E., Martin, N., Prevot, L., et al. 1983, A\&AS, 51, 277

Reid, N., Glass, I. S., \& Catchpole, R. M. 1988, MNRAS, 232, 53

Reid, N., Tinney, C., Mould, J. 1990, ApJ, 348, 98

Reynolds, A. P., Parmar, A. N., Hakala, P. J., et al. 1999, A\&AS, 134, 287

Sasaki, M., Haberl, F., \& Pietsch, W. 2000, A\&AS, 147, 75 [SHP00]

Schmidt, M., Hasinger, G., Gunn, J., et al. 1998, A\&A, 329, 495

Schmidt-Kaler, T., Gochelmann, J., Österreicher, M. O., et al. 1999, MNRAS, 306, 279

Schmidtke, P. C., Cowley, A. P., Crane, J. D., et al. 1999, AJ, 117,927

Smith, R. C., Chu, Y.-H., Mac Low, M.-M., et al. 1994, AJ, 108,1266

Stanimirovic, S., Staveley-Smith, L., Dickey, J. M., et al. 1999, MNRAS, 302, 417

Supper, R., Hasinger, G., Lewin, W. H. G., et al. 2001, A\&A, 373,63

Thompson, R. J., Shelton, R. G., \& Arning, C. A. 1998, AJ, 115,258

van den Bergh, S. 2000, PASP, 112, 529

Voges, W., Aschenbach, B., Boller, Th., et al. 2000, ROSAT All-Sky Survey Faint Source Catalog, Vizier On-line Data Catalog IX/29

Wang, Q., Hamilton, T., Helfand, D. J., \& Wu, X. 1991, ApJ, 374,475

Westerlund, B. E., Olander, N., \& Hedin, B. 1981, A\&AS, 43, 267

Will, J.-M., Bomans, D. J., Tucholke, H.-J., et al. 1995, A\&AS, 112,367

Wright, A. E., Griffith, M. R., Burke, B. F., \& Ekers, R. D. 1994, ApJS, 91, 111

Yokogawa, J., Imanishi, K., Tsujimoto, M., et al. 2000, ApJ, 128,491

Zimmermann, H. U., Becker, W., Belloni, T., et al. 1994, MPE Report, 257 